\title{
Microarray analysis of the time-dependent expression profiles of long non-coding RNAs in the progression of vein graft stenotic disease
}

\author{
JIAYU SHEN ${ }^{*}$, HONGWEI ZHANG* , CHEN LU* ${ }^{*}$ JUN GU, YU ZHANG and JIA HU \\ Department of Cardiovascular Surgery, West China Hospital, Sichuan University, Chengdu, Sichuan 610041, P.R. China
}

Received September 12, 2020; Accepted March 11, 2021

DOI: $10.3892 /$ etm.2021.10067

\begin{abstract}
Long non-coding RNAs (lncRNAs) have been reported to be involved in various biological processes, including cell proliferation and apoptosis. However, the expression profiles of lncRNAs in patients with vein graft restenosis remain unknown. In the present study, the time-dependent expression profiles of genes in vein bypass grafting models were examined by microarray analysis. A total of 2,572 lncRNAs and 1,652 mRNAs were identified to be persistently significantly differentially expressed. Gene Ontology and Kyoto Encyclopedia of Genes and Genomes enrichment analysis was performed to investigate the functions of these lncRNAs. A total of 360 lncRNAs and 135 protein-coding genes were predicted to be involved in the vascular remodeling process. Co-expression network analysis revealed the association between 194 lncRNAs and seven associated protein-coding genes, including transforming growth factor- $\beta 1$, Fes, Yes1 associated transcriptional regulator, sphingosine-1-phosphate receptor $1, \mathrm{Src}$, insulin receptor and melanoma cell adhesion molecule. Moreover, reverse transcription-quantitative PCR results supported those of the microarray data, and overexpression of AF062402, which regulates the transcription of Src, stimulated the proliferation of primary vascular smooth muscle cells. The findings of the present study may facilitate the development of novel therapeutic targets for vein graft
\end{abstract}

Correspondence to: Dr Jia Hu, Department of Cardiovascular Surgery, West China Hospital, Sichuan University, 37 GuoXue Alley, Chengdu, Sichuan 610041, P.R. China

E-mail: humanjia@msn.com

*Contributed equally

Abbreviations: lncRNAs, long non-coding RNAs; GO, Gene Ontology; KEGG, Kyoto Encyclopedia of Genes and Genomes; VSMCs, vascular smooth muscle cells; CABG, Coronary artery bypass grafting; S1P, sphingosine-1-phosphate; CCK-8, Cell Counting Kit-8; RT-qPCR, reverse transcription-quantitative PCR

Key words: coronary artery bypass grafting; co-expression network; long non-coding RNAs; mRNAs; microarray analysis; vein graft stenotic disease restenosis and may help to improve the prognosis of patients following coronary artery bypass grafting.

\section{Introduction}

Coronary artery bypass grafting (CABG) remains the primary treatment for patients with unprotected left main and multi-vessel coronary artery disease (1). Although total arterial revascularization has been advocated, the autologous saphenous vein is still the most commonly used graft owing to its availability and length (2). However, the per-graft occlusion rate of the vein graft is estimated to be up to $25 \%$ during the first 12 months following $\mathrm{CABG}$, and almost half of the venous conduits fail at 10 years (3).

Clinically, vein graft stenotic disease leads to considerable post-operative adverse events, including recurrent angina, myocardial infraction and repeat revascularization (4). To date, the pathophysiological mechanisms underlying venous wall remodeling remain to be fully understood. Thrombosis, neointimal hyperplasia and superimposed atherosclerosis are generally regarded as primary causes of vein graft failure that have attracted the most research interest for therapeutic applications (5). To the best of our knowledge, however, apart from statins and antiplatelet agents, no other interventions have proven to be clinically effective (6). Considering the aging population and the persistent increase in the incidence of patients with CABG living with symptomatic vein graft stenotic disease in Europe and USA (7-9), further identification of novel biomarkers that can be effectively targeted is urgently required (10).

There has been an increasing interest in the role of non-coding RNAs in the pathogenesis of vascular disease (11). Based on transcript size, non-coding RNAs are classified into small, medium and long non-coding RNAs (lncRNAs) (12). The biogenesis and functional properties of small RNAs, particularly microRNAs (miRNAs or miRs), in the onset and progression of cardiovascular disease have been well-described (13). For example, the administration of miR-221 has been shown to effectively inhibit endothelial cell proliferation at predilection sites (14). However, the expression profiles and potential functions of IncRNAs in the progression of venous wall remodeling following bypass grafting remain unknown. The present study aimed to investigate the dynamic expression profiles of lncRNAs following bypass grafting in a 
rat model, and to elucidate the potential association between lncRNAs and vein graft stenotic disease.

\section{Materials and methods}

Rat model establishment. A total of 12 male Sprague Dawley rats (age, 6-8 weeks) were purchased from the Laboratory Animal Center of Sichuan University (Chengdu, Sichuan, China). They were randomly divided into four groups to ensure equal weight distribution (weight, $248 \pm 27 \mathrm{~g}$ ). One group receiving sham surgery was set as the control (day 0; $\mathrm{n}=3$ ), whilst the other three groups were set as the experimental groups according to different time points after vein bypass grafting (7, 14 and 28 days after surgery, respectively; $n=3$ per group). For the experimental groups, the rats were anesthetized with intraperitoneal injection of $10 \%$ chloral hydrate (350 mg/kg). Heparin (100 U/kg) and cefazolin $(50 \mathrm{mg} / \mathrm{kg})$ were administered intraperitoneally for anticoagulation and infection prevention, respectively. No signs of peritonitis were observed. A length of vein graft $(1 \mathrm{~cm})$ was harvested from the external jugular vein and preserved in heparinized saline $(25 \mathrm{U} / \mathrm{ml})$. The vein was subsequently grafted to the carotid artery (Fig. 1). For the control group, all the procedures performed were identical with those in the experimental groups except vein bypass grafting was not peroformed. These rats were housed in a temperature-controlled room at $20 \pm 2^{\circ} \mathrm{C}$ and relative humidity of $50 \%$, with a 12 -h light/dark cycle. Free access to food and water was provided. These experiments were performed according to the National Institutes of Health Guideline for the Care and Use of Laboratory Animals (15). The present study was approved by the Animal Ethical and Welfare of Sichuan University, Sichuan, China (approval no. 2018103A).

Total RNA extraction and array hybridization. Following the intraperitoneal administration of $10 \%$ chloral hydrate $(350 \mathrm{mg} / \mathrm{kg}$ ) for anesthetization, the rats were sacrificed by cervical dislocation. Vein samples $(\sim 1 \mathrm{~cm})$ were harvested from the different groups. Total RNA was isolated using TRIzol ${ }^{\circledR}$ reagent according to the manufacturer's instructions (Invitrogen; Thermo Fisher Scientific, Inc.). The purity of the RNA samples was analyzed using the Nanodrop spectrophotometer (Implen $\mathrm{GmbH}$ ), which measured absorbance at 230,260 and $280 \mathrm{~nm}$. The 260/280 and 260/230 absorbance ratios were calculated to evaluate $\mathrm{RNA}$ purity $\left(\mathrm{A}_{260} / \mathrm{A}_{280}\right.$ ratios are 1.8-2.2 and $\mathrm{A}_{260} / \mathrm{A}_{230}$ ratios are over 1.7). 1.0\% non-denaturing agarose gel electrophoresis was used to determine the RNA integrity. The microarray analysis was performed by Kangchen BioTech Co., Ltd. using Agilent Technologies Rat LncRNA Microarray scanner (cat no. G2505C; Agilent Technologies, Inc.). Sample labeling and microarray hybridization were performed according to the manufacturer's protocols. In brief, ribosomal RNA was removed from total RNA using a mRNA-ONLY ${ }^{\mathrm{TM}}$ Eukaryotic mRNA Isolation kit (cat. no. MOE51010; Zhongbei Linge Biotechnology, Ltd.; http://www.bjzblg.com/product/18395.html). The remaining RNA was amplified and reverse transcribed into fluorescent complementary (c)RNA using the PrimeScript ${ }^{\mathrm{TM}}$ RT Reagent Kit (cat. no. RR037B; Takara bio, Inc.) at $37^{\circ} \mathrm{C}$ for $15 \mathrm{~min}$, followed by $85^{\circ} \mathrm{C}$ for $5 \mathrm{sec}$. The RNeasy Mini kit and NanoDrop ND-1000 instrument were used to purify and measure the concentration of the labeled cRNAs. A total of $5 \mu \mathrm{l} 10 \mathrm{X}$ Blocking Agent and $1 \mu 1$ 25X Fragmentation Buffer were mixed with $1 \mu \mathrm{g}$ labeled cRNA and the mixture was then heated at $60^{\circ} \mathrm{C}$ for $30 \mathrm{~min}$. Subsequently, $25 \mu 12 \mathrm{X}$ GE Hybridization buffer was added to dilute the labeled cRNA. The hybridization solution was incubated for $17 \mathrm{~h}$ at $65^{\circ} \mathrm{C}$ in an Agilent Technologies Hybridization oven. After washing, the hybridized arrays were scanned with using an Agilent microarray scanner (cat no. G2505C; Agilent Technologies, Inc.).

Bioinformatics analysis of microarray data. Raw data were extracted from the acquired array images using Agilent Feature Extraction software 11.0.1.1 (Agilent Technologies, Inc.). GeneSpring GX v12.1 software (Agilent Technologies, Inc.) was used to normalize quantiles of raw data. Genes with an adjusted $\mathrm{P}$-value $<0.01$ and an absolute value of $\log _{2}$ (fold change) $>2.0$ were considered to be significantly differentially expressed. Hierarchical clustering analysis was performed to observe the different clustering patterns of 1ncRNAs and mRNAs between the control and experimental groups. Scatter and volcano plots analysis were constructed to express the differentially expressed genes. In order to examine the distribution of differentially expressed lncRNAs, histograms and a pie chart were generated to reflect the chromosomal distribution, lengths and regulation mechanisms of the differentially expressed lncRNAs.

Prediction and functional enrichment analysis of IncRNA target genes. The protein-coding genes targeted by lncRNAs were predicted via $c e$ - and trans-regulation analyses, respectively. In the $c e$-regulation analysis, IncRNAs compete with shared miRNAs to regulate target protein-coding genes, which are genomically neighboring within $10 \mathrm{~kb}$. In the trans-regulation analysis, lncRNAs regulate protein-coding genes sequences matched with low complement energy (inclusion criteria, G<-20). Kyoto Encyclopedia of Genes and Genomes (KEGG) and Gene Ontology (GO) enrichment analyses were performed to evaluate the functional pathways and biological relevance of the lncRNA co-expressed mRNAs using Database for Annotation, Visualization and Integrated Discovery (david.abcc.ncifcrf.gov/). All functional analyses were performed using Enrichr (16).

Co-expression network analysis. The co-expression of lncRNAs and protein-coding genes was assessed using Gephi 0.9.2 (17) and Pearson's correlation test $(\mathrm{P}<0.05)$. Upregulated lncRNAs were presented as orange nodes and downregulated IncRNAs were presented as blue nodes. Protein-coding genes targeted by 1 chNAs were presented as green nodes.

Verification of microarray data by reverse transcriptionquantitative $(R T-q) P C R$. RT-qPCR was performed to validate the microarray data. Samples were divided into control group and experimental groups. Samples of control group were collected from rats receiving sham surgery $(n=3)$, samples of experimental groups were collected from rats that received vein bypass grafting (7, 14 and 28 day after surgery, respectively; $n=3$ per group). Total RNA was 


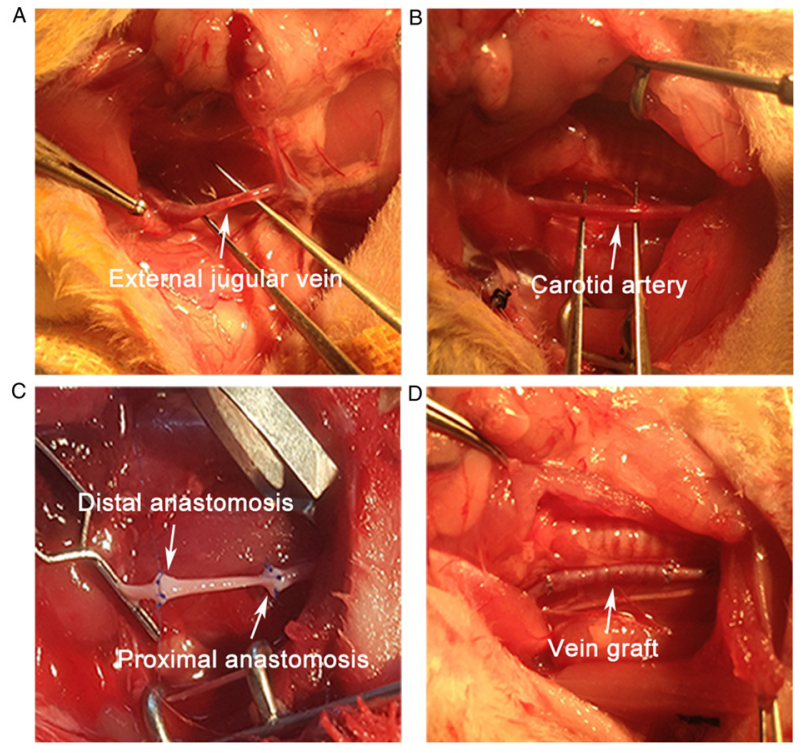

Figure 1. Establishment of an external jugular vein carotid bypass rat model. (A) Exposure of the external jugular vein; (B) Exposure of the carotid artery; (C) The external jugular vein was anastomosed to the carotid artery; (D) The blood flow in the graft was patent.

extracted with TRIzol ${ }^{\circledR}$ reagent from the remaining portion of tissues not used for the lncRNA microarray. Subsequently, first-strand complementary DNAs were generated using SuperScript ${ }^{\mathrm{TM}}$ III Reverse Transcriptase (Invitrogen; Thermo Fisher Scientific, Inc.) following the manufacturer's protocols. The RT-qPCR process was performed using a ViiA-7 RT-PCR System (Applied Biosystems; Thermo Fisher Scientific, Inc.) and Arraystar SYBR-Green Real-time qPCR Master Mix (cat no. AS-MR-005-25; Arraystar, Inc.). The reactions were performed under the following conditions: Initial denaturation at $95^{\circ} \mathrm{C}$ for $30 \mathrm{sec}$, followed by 40 cycles of $95^{\circ} \mathrm{C}$ for $5 \mathrm{sec}$ and $60^{\circ} \mathrm{C}$ for $30 \mathrm{sec}$. The relative expression levels of lncRNAs were quantified using the $2^{-\Delta \Delta \mathrm{Cq}}$ method (14). $\beta$-actin served as an internal control. The primers for RT-qPCR were designed according to the lncRNA sequences in NONCODE (version v6.0; http://www.noncode.org) and primer sequences were synthesized and purified by Kangchen BioTech Co., Ltd. The primer sequences are presented in Table I. All experiments were performed in triplicate for error reduction.

Cells and cell culture. After the rats which were used for lncRNA microarray were sacrificed by cervical dislocation, primary vascular smooth muscle cells (VSMCs) were obtained from normal external jugular vein. All procedures were performed according to a previously published protocol (18). In brief, external jugular vein was isolated from adherent tissue, and adventitia and endothelium were denuded. Pieces of vein $\left(1 \mathrm{~mm}^{2}\right)$ were cultured in serum-free DMEM/F12 (Gibco; Thermo Fisher Scientific, Inc.) supplemented with 20\% FBS (Gibco; Thermo Fisher Scientific, Inc.) and 1\% penicillin-streptomycin at $37^{\circ} \mathrm{C}$ in a $5 \% \mathrm{CO}_{2}$ humidified incubator. Cells migrated from the sections of aorta after 7-9 days. Vein pieces were then removed and VSMCs were left to proliferate. Experiments used VSMCs from passages 3-6 at 80-90\% confluence.
Infection with lentiviral vector and cell proliferation assay. VSMCs suspensions were seeded into six-well plates at a density of $4 \times 10^{5}$ cells/well. DMEM/F12 (Gibco; Thermo Fisher Scientific, Inc.) with $10 \%$ v/v FBS (Gibco; Thermo Fisher Scientific, Inc.) was removed the following day and replaced with virus concentrated liquid (Shanghai OBiO Technology Co., Ltd.). Stable overexpression of Src was achieved by construction of the GL107 pSLenti-EF1-EGFPP2A-Puro-CMV-MCS-3xFLAG-WPRE lentivirus (Shanghai OBiO Technology Co., Ltd.), containing the AF062402, one of the significantly upregulated lncRNAs, gene vector with green fluorescence protein (GFP; Shanghai OBiO Technology Co., Ltd.) to detect transfection efficacy. Polybrene reagent (Shanghai OBiO Technology Co., Ltd.) was diluted to a final concentration of $5 \mu \mathrm{g} / \mathrm{ml}$. Multiplicity of infection (MOI) values $(1,2,5,10,30$ and 50) were then tested on VSMCs. A total of $4 \times 10^{6}$ lentivral particles were used for transfection and the optimal MOI value was 10 in the present study. At this value, the efficiency of infection reached $80 \% 3$ days post-infection. After swirling the plate gently to mix the cells, the plate was placed in an incubator with $5 \% \mathrm{CO}_{2}$ at $37^{\circ} \mathrm{C}$ for $24 \mathrm{~h}$. After $24 \mathrm{~h}$, the virus concentrated medium was removed and replaced by DMEM/F12 medium (Gibco; Thermo Fisher Scientific, Inc.) with 10\% FBS (Gibco; Thermo Fisher Scientific, Inc.). At 3 days post-transfection, GFP expression was observed as the lentivirus was integrated into the VSMC genome, in five randomly-selected fields using a fluorescence microscope (magnification, x200). Cells with a transfection efficiency $>80 \%$ on day 3 were selected for subsequent analysis. VSMCs transfected with AF062402 lentiviral expression vector were set as the AF062402 intervention group. Non-transfected cells were set as the blank control and lentivirus without the AF062402 gene was transfected as the negative control (NC). At $72 \mathrm{~h}$ following transfection, RT-qPCR was performed to verify the expression levels of AF062402 in the different groups. Moreover, Cell Counting Kit-8 (CCK-8) assay was performed using a kit from Abcam. VSMCs were digested into single cell suspensions and seeded into 96 -well plates $\left(5 \times 10^{3}\right.$ cells $\left./ \mathrm{ml}\right)$. After $48 \mathrm{~h}, \mathrm{CCK}-8$ solution $(10 \mu \mathrm{l} /$ well) was added and the plates were incubated for $2.5 \mathrm{~h}$ at $37^{\circ} \mathrm{C}$ before the optical density (OD) value was detected at $460 \mathrm{~nm}$ using a plate reader. All experiments were performed in triplicate.

Statistical analysis. Feature Extraction software (Agilent Technologies, version 11.0.1.1) was used to analyze the acquired array images. Differentially expressed lncRNAs and mRNAs were identified by adjusted P-value $<0.01$ and absolute value of the $\log _{2}$ (fold change) $>2.0$. The results of CCK-8 assay and RT-qPCR are expressed as the mean \pm SD $(n=3)$. Statistical comparisons between groups were performed using one-way analysis of variance with Tukey's post hoc multiple comparison test. $\mathrm{P}<0.05$ was considered to indicate a statistically significant difference.

\section{Results}

Overview of microarray data and expression patterns of lncRNAs. A total of 8,041 lncRNAs and 13,001 mRNAs were detected. Box plots and hierarchical clustering 
Table I. PCR primers used for expression analysis.

\begin{tabular}{ll}
\hline Gene & \multicolumn{1}{c}{ Primer sequence $\left(5^{\prime} \rightarrow 3^{\prime}\right)$} \\
\hline AF062402 & F: TGGCCCAGAACGGAAATATCA \\
& R: ACTAGCCCGGAGTTTGACCAT \\
BC091437 & F: GGCACCAGAGCTACCATTCC \\
& R: CGTGGACTTGGCAGGAGAAA \\
BC166461 & F: GCACAAGGTTCAAAAAGGCAA \\
& R: GCTTGGTAGCCATACTTCTGGAA \\
Src & F: GAACCCGAGAGGGACCTTC \\
& R: GAGGCAGTAGGCACCTTTTGT \\
Edg1 & F: ATGGTGTCCACTAGCATCCC \\
& R: CGATGTTCAACTTGCCTGTGTAG \\
Mcam & F: CCCAAACTGGTGTGCGTCTT \\
& R: GGAAAATCAGTATCTGCCTCTCC \\
F-actin & F: CGAGTACAACCTTCTTGCAGC \\
& R: ACCCATACCCACCATCACAC
\end{tabular}

F, forward; R, reverse; Mcam, melanoma cell adhesion molecule.

analysis (Fig. S1) provided an overview of all normalized genes in all samples. As shown in the hierarchical clustering analyses, scatter and volcano plots, distinct expression profiles of lncRNAs and mRNAs at different post-operative time points were observed (Figs. $2 \mathrm{~A}$ and S2). The lncRNAs were then classified based on chromosomal distribution and length (Fig. 2B and C). Subsequently, 13 lncRNA profiles with statistical significance were selected for further investigation (Fig. 2D). A total of 2,572 lncRNAs and 1,652 associated mRNAs with significant changes in expression levels $(\mathrm{P}<0.01$; fold change $>2$ ) were detected. IncRNAs were categorized into five subgroups (Fig. 2E) according to their association with adjacent genes as follows: i) Intergenic lncRNAs, $27.17 \%$; ii) exonic-antisense and exonic-sense lncRNAs, 5.65 and $23.66 \%$, respectively; iii) intronic-antisense and intronic-sense lncRNAs, 6.64 and $9.28 \%$, respectively; iv) lncRNAs transcribed on the opposite strand of a coding gene, $1.90 \%$ and $v$ ) the remaining lncRNAs transcribed in other patterns, $27.29 \%$. The numbers of IncRNAs with significantly upregulated or downregulated expression at different post-operative time points are presented in Fig. 2F. Moreover, the top 10 lncRNAs with significant changes in expression levels at different post-operative time points are presented in Table II.

IncRNA target prediction and functional annotation. In order to identify the potential functional pathways of lncRNAs with significant changes in expression levels, $c$ - and trans-regulation analysis results were combined and overlapping mRNAs were selected for further 'GO' and 'KEGG' analyses. 'GO' analysis revealed a significant enrichment of 4,993 'GO' terms $(\mathrm{P}<0.01)$, of which 'cellular process', 'protein binding' and 'cell/cell part' were the top-listed terms involved in biological process, molecular function and cell component, respectively (Fig. 3A). 'KEGG' analysis revealed that 298 biological pathways were enriched $(\mathrm{P}<0.01)$, of which several were associated with vascular remodeling, including 'cell adhesion molecules', 'platelet activation' and 'vascular smooth muscle contraction' (Fig. 3B). Among these, nine biological processes associated with vascular remodeling process were selected for further analysis, including 'cell adhesion', 'cell migration', 'cell proliferation', 'MAP kinase activation', 'platelet activation', 'chemotaxis', 'WNT protein secretion', 'stimulation responses' and 'growth factor activity'. The changes in the numbers of IncRNAs in these selected biological processes are presented in Fig. 4A.

Co-expression network analysis of IncRNAs and associated protein-coding genes. Based on the results of functional analysis, a total of $360 \mathrm{lncRNAs}$ and 135 associated protein-coding genes were predicted to be involved in the vascular remodeling process (Table SI). Among these, 194 lncRNAs and seven previously reported associated protein-coding genes (19-32) involved in mediating vascular disease were selected to construct a co-expression network. Each protein-coding gene was targeted by multiple lncRNAs (Fig. 4B).

$R T$-qPCR validation and stimulation of VSMC proliferation via overexpression of AF062402. A total of three lncRNAs and associated protein-coding genes were randomly selected to verify the microarray data by RT-qPCR. AF062402 and Src were upregulated, whereas BC091437 and Edg1 were downregulated (Fig. 5A). Moreover, the upregulation of BC166461 was accompanied by downregulation of melanoma cell adhesion molecule (Mcam). The relative expression levels of the selected lncRNAs and associated protein-coding genes were consistent with the microarray data. Moreover, RT-qPCR confirmed that the relative expression of AF062402 in the Lentivirus-AF062402 group was significantly higher than that in the blank and Lentivirus-NC groups. The overexpression of AF062402 stimulated the proliferation of primary VSMCs compared with the other two groups (Fig. 5B). 
A
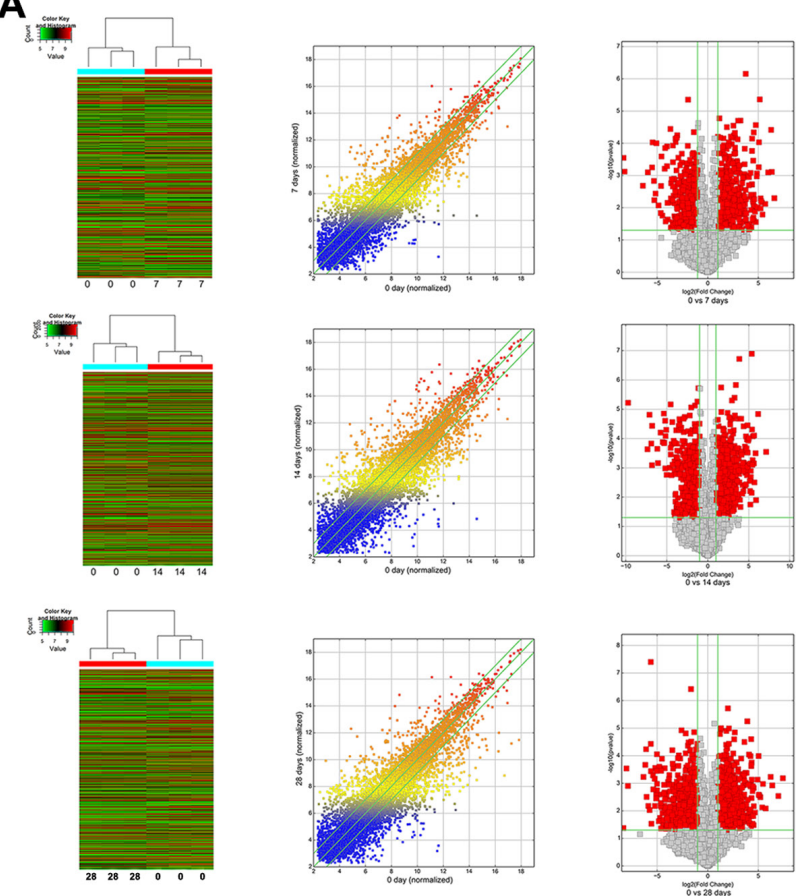

D
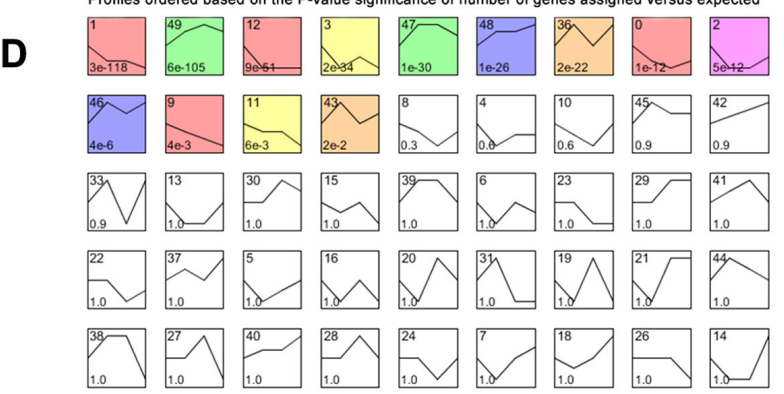

E
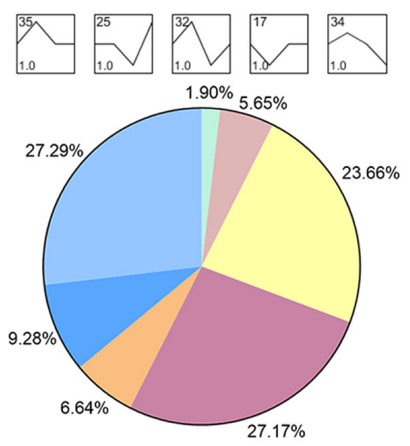

$\square$ bidirectional

$\square$ exonic-antisense

$\square$ exonic-sense

$\square$ intergenic

$\square$ intronic-antisense

$\square$ intronic-sense

$\square$ other

Total $=\mathbf{2 5 7 2}$
B
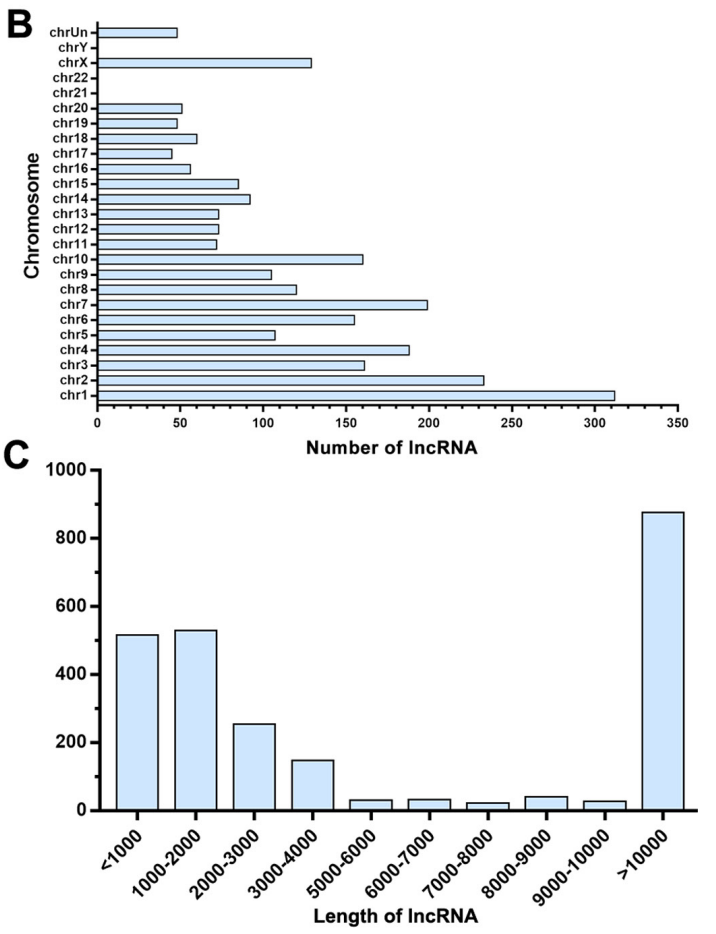

$\mathbf{F}$
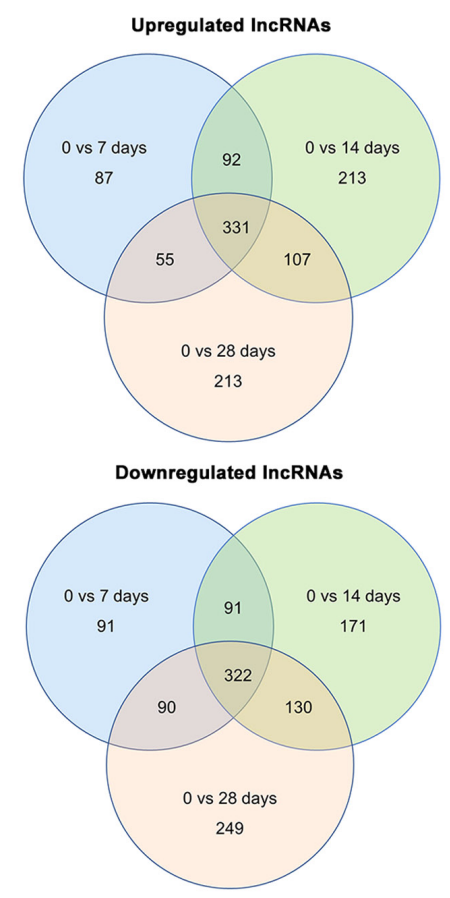

Figure 2. Overview of significantly differentially expressed lncRNAs. (A) Heatmaps showing lncRNAs that were differentially expressed between groups. Scatter and volcano plots illustrate variations in lncRNA expression between different time points (0 vs. days 7, 14 and 28). (B) Chromosomal and (C) length distribution of differentially expressed lncRNAs. (D) Time series analysis revealed the temporal expression patterns of differentially expressed lncRNAs. Each box represents the time-dependent expression profile of a lncRNA. Upper value refers to the number of profiles; lower value indicates P-value of the profiles in each box. (E) Pie chart illustrating the classification of differentially expressed lncRNAs based on genomic location relative to neighboring or overlapping genes. (F) Time-dependent change in the number of differential expressed lncRNAs at three post-operative timepoints. IncRNAs, long non-coding RNAs.

\section{Discussion}

Vein graft restenosis is a primary long-term complication which leads to a poor prognosis of patients with CABG (10). However, apart from statins and antiplatelet agents, few therapeutic strategies have been proven to have significant clinical efficacy (6). Multiple studies have demonstrated that lncRNAs serve key roles in the pathophysiology of various diseases by participating in numerous regulatory processes, such as DNA methylation, histone modification, basal transcription, post-transcriptional processes and protein binding $(33,34)$, indicating that lncRNAs may be potential intervention targets for vein graft restenosis.

The present study systemically screened a genome-wide microarray profile of lncRNA and mRNA expression at different time points following the establishment of rat autogenous vein bypass grafts. A total of $8,041 \mathrm{lncRNAs}$ 
Table II. Top 10 significantly differential expressed lncRNAs at days 7, 14 and 28 vs. day 0 .

A, Day 7

\begin{tabular}{|c|c|c|c|c|c|}
\hline \multicolumn{3}{|c|}{ Upregulated } & \multicolumn{3}{|c|}{ Downregulated } \\
\hline $\operatorname{lncRNA}$ & P-value & $\mathrm{FC}$ & lncRNA & P-value & $\mathrm{FC}$ \\
\hline MRAK132609 & 0.0050 & 95.50 & XR_006421 & 0.0003 & 320.62 \\
\hline EF199812 & 0.0008 & 79.79 & ВC086433 & 0.0008 & 298.86 \\
\hline MRAK142484 & 0.0000 & 76.34 & MRuc008egb & 0.0021 & 85.27 \\
\hline BC158825 & 0.0008 & 62.77 & XR_008627 & 0.0008 & 60.58 \\
\hline AJ005396 & 0.0076 & 51.12 & BC169049 & 0.0006 & 45.32 \\
\hline MRAK005319 & 0.0069 & 40.62 & AB049626 & 0.0001 & 43.74 \\
\hline XR_007646 & 0.0061 & 38.35 & MRuc0081zo & 0.0015 & 39.94 \\
\hline X89963 & 0.0051 & 37.14 & MRAK046251 & 0.0058 & 38.96 \\
\hline MRuc007pas & 0.0000 & 35.18 & MRAK005337 & 0.0036 & 35.81 \\
\hline BC167061 & 0.0001 & 33.34 & BC 166501 & 0.0001 & 33.57 \\
\hline
\end{tabular}

B, Day 14

\begin{tabular}{|c|c|c|c|c|c|}
\hline \multicolumn{3}{|c|}{ Upregulated } & \multicolumn{3}{|c|}{ Downregulated } \\
\hline lncRNA & $\mathrm{P}$-value & $\mathrm{FC}$ & lncRNA & P-value & $\mathrm{FC}$ \\
\hline EF199812 & 0.0003 & 138.51 & ВC086433 & 0.0000 & 848.08 \\
\hline MRAK132609 & 0.0000 & 70.79 & XR_008627 & 0.0002 & 164.20 \\
\hline MRAK142484 & 0.0003 & 56.92 & AJ517196 & 0.0000 & 138.75 \\
\hline XR_007646 & 0.0031 & 51.58 & MRAK005337 & 0.0001 & 118.56 \\
\hline BC167061 & 0.0001 & 50.03 & XR_006421 & 0.0008 & 112.10 \\
\hline MRAK143033 & 0.0007 & 49.40 & MRuc008egb & 0.0000 & 110.95 \\
\hline BC158675 & 0.0001 & 44.93 & MRuc0081zo & 0.0002 & 71.61 \\
\hline XR_008202 & 0.0005 & 44.29 & BC169049 & 0.0007 & 67.66 \\
\hline EF673689 & 0.0008 & 42.76 & AB049626 & 0.0001 & 54.08 \\
\hline BC158638 & 0.0000 & 42.24 & MRuc007smc & 0.0001 & 45.26 \\
\hline
\end{tabular}

C, Day 28

\begin{tabular}{|c|c|c|c|c|c|}
\hline \multicolumn{3}{|c|}{ Upregulated } & \multicolumn{3}{|c|}{ Downregulated } \\
\hline lncRNA & P-value & $\mathrm{FC}$ & $\operatorname{lncRNA}$ & P-value & FC \\
\hline MRAK132609 & 0.0007 & 170.96 & XR_006421 & 0.0003 & 262.57 \\
\hline MRAK020463 & 0.0029 & 125.46 & XR_006359 & 0.0012 & 237.66 \\
\hline AJ005396 & 0.0012 & 82.50 & MRAK149123 & 0.0006 & 82.12 \\
\hline X89963 & 0.0006 & 74.27 & MRAK084152 & 0.0002 & 72.11 \\
\hline NR_027324 & 0.0082 & 52.56 & BC086433 & 0.0001 & 68.54 \\
\hline EF199812 & 0.0011 & 43.63 & MRAK148606 & 0.0031 & 65.84 \\
\hline AF130879 & 0.0075 & 41.29 & MRAK037975 & 0.0067 & 58.59 \\
\hline MRAK164133 & 0.0032 & 36.60 & XR_007815 & 0.0094 & 53.91 \\
\hline L38717 & 0.0027 & 33.82 & MRAK080917 & 0.0000 & 49.53 \\
\hline U57362 & 0.0026 & 32.63 & MRAK080330 & 0.0000 & 49.43 \\
\hline
\end{tabular}

FC, fold change; lncRNAs, long-noncoding RNAs.

and 13,001 mRNAs were detected. In total, 2,572 dysregulated IncRNAs were screened following vein bypass grafting and lncRNA-associated protein coding genes were predicted by $c e$ - and trans-regulation analysis. Through 
A
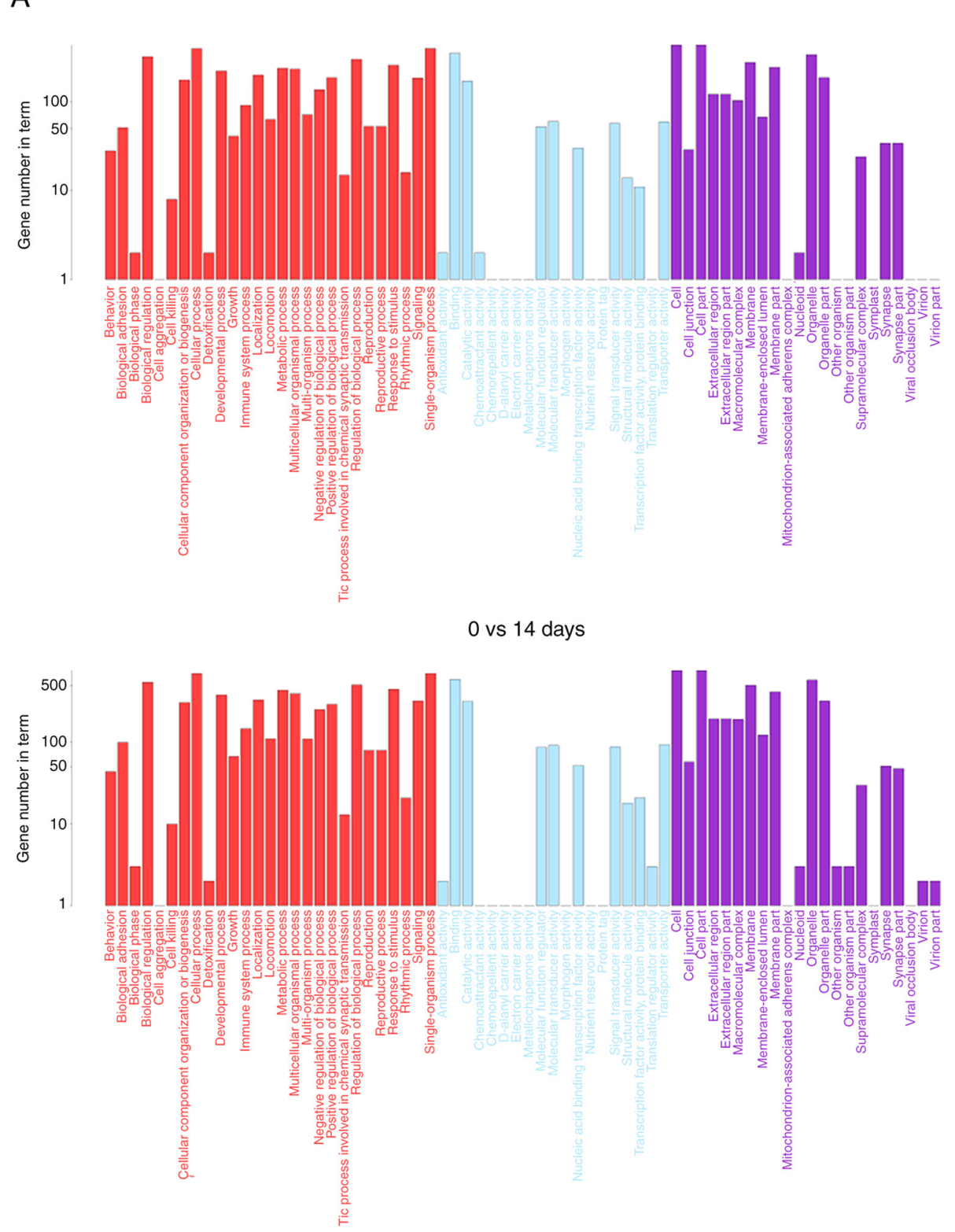

0 vs 28 days

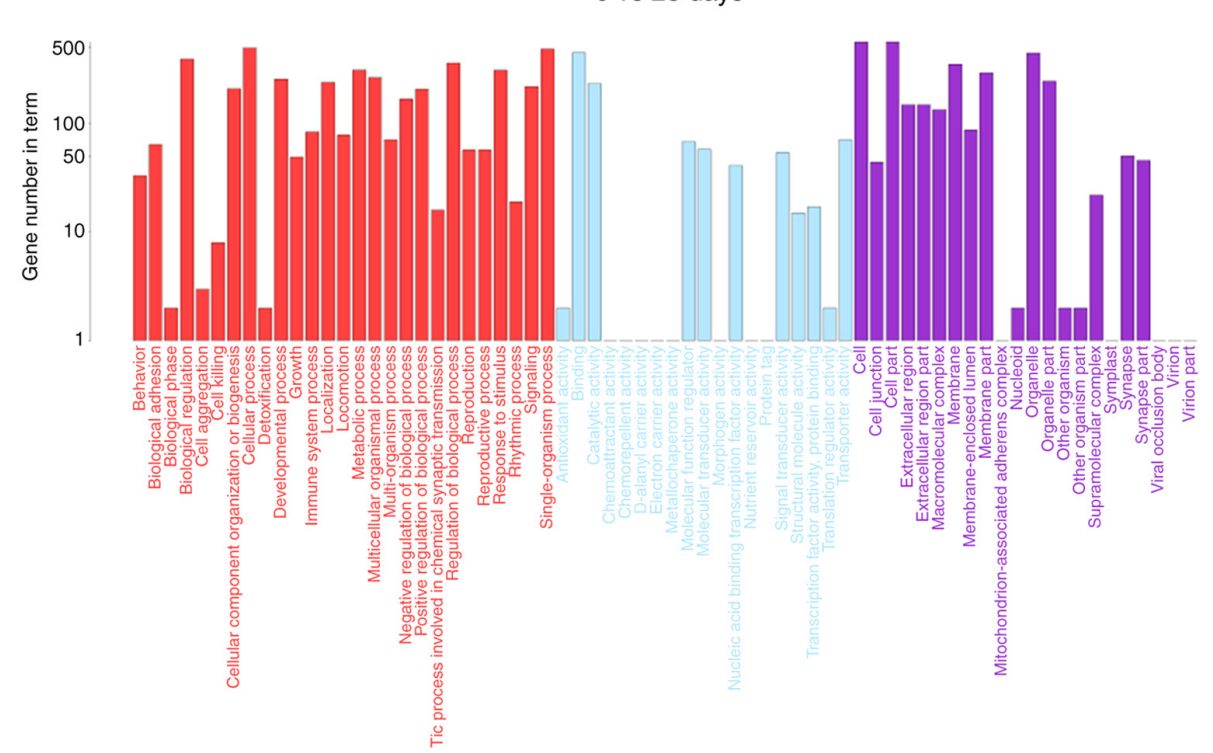

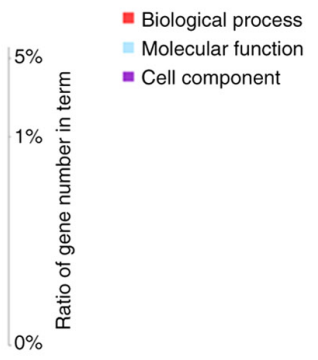

- Bological process
$5 \%$ Molecular function

$1 \%$ - Molecular function
- Cell component

(1)

$0 \%$

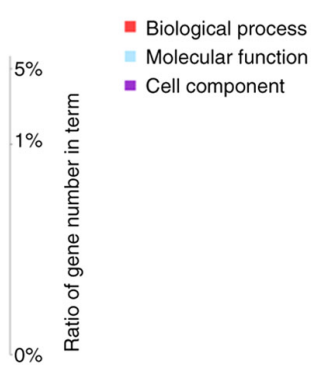

Figure 3. Continued. 
B
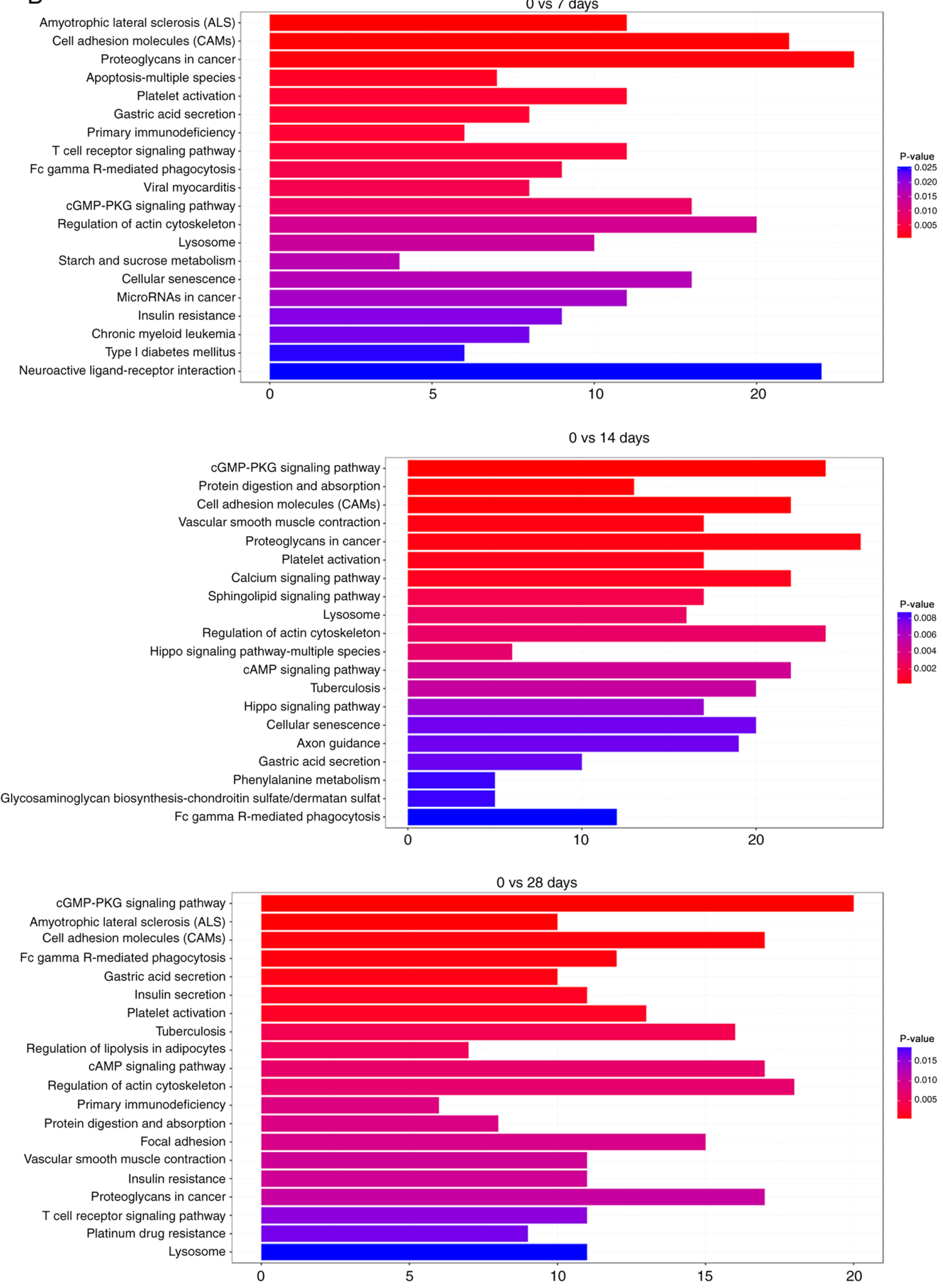

Figure 3. 'GO' and 'KEGG' pathway enrichment analysis of lncRNA-associated protein-coding genes. (A) Significant BP, MF and CC terms in 'GO' enrichment analysis. (B) 'KEGG' enrichment analysis of significantly enriched pathways. P<0.05. GO, Gene Ontology; KEGG, Kyoto Encyclopedia of Genes and Genomes; lncRNAs, long non-coding RNAs; BP, biological process; MF, molecular function; CC, cell component.

enrichment analysis, nine biological processes associated with the vascular remodeling process were selected. Moreover, co-expression network analysis revealed an association between IncRNAs and their target genes, which were reported to play roles in mediating vascular disorders (19-32). Furthermore, it was found that AF062402, one of the significantly upregulated lncRNAs, may serve a role in inducing vein graft restenosis.

Various pathophysiological changes secondary to vascular bypass grafting, such as inflammation, platelet activation, stimulation responses, leukocyte chemotaxis and mechanical intimal injury, are regarded as primary causes of 

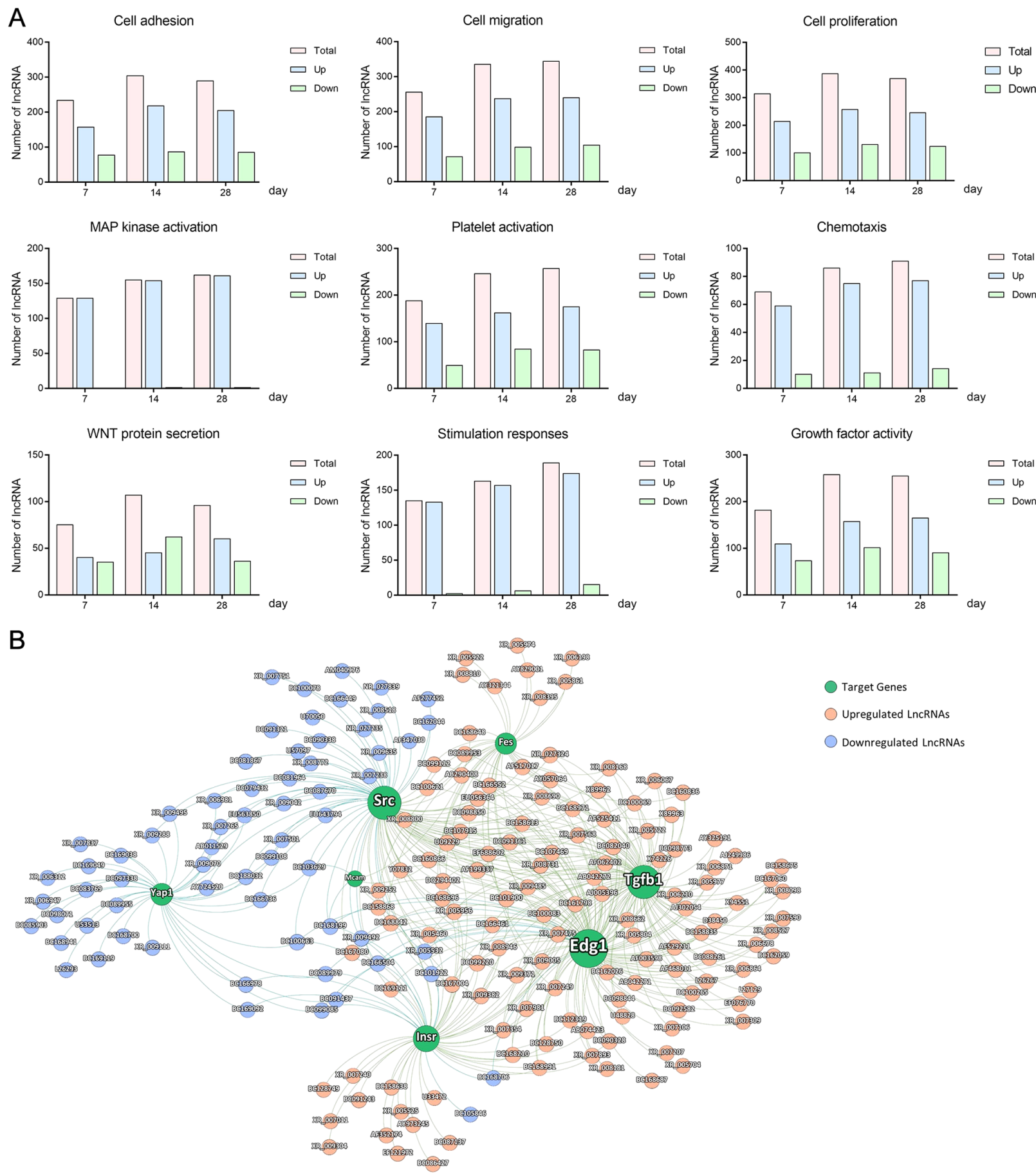

Figure 4. Biological processes associated with vascular remodeling and the interaction network between selected lncRNAs and associated protein-coding genes. (A) Time-dependent change in the number of differentially expressed lncRNAs predicted to be involved in 'cell adhesion', 'migration', 'cell proliferation', 'MAP kinase activation', 'platelet activation', 'chemotaxis', 'WNT protein secretion', 'stimulation responses' and 'growth factor activity'. (B) Co-expression network between selected lncRNAs and associated protein-coding genes. Orange and blue circles indicate upregulated and downregulated lncRNAs, respectively. Green circles indicate protein-coding genes overlapped with mRNAs. lncRNA, long non-coding RNA.

vein graft restenosis (35-37). The inflammatory and thrombotic cascade responsible for early graft thrombosis and failure can be caused by direct endothelial injury or endothelial activation (38). Intimal hyperplasia and atherosclerosis are also considered to be primary causes of lumen remodeling of the graft, which are associated with the processes of leukocyte adhesion and vascular endothelial cells and VSMC proliferation (35). Several studies have implicated the production of reactive oxygen species in disease progression in grafts $(39,40)$. There is increasing evidence to demonstrate a pivotal role of MAPK activation cascades in vascular restenosis, which contribute to proliferation of VSMCs and neointima formation via downstream proteins (41). It is also known that leukocyte chemotaxis serves a key role in a complex mechanism, involving an association between inflammation factors, platelets and vascular disease (36). Moreover, exposure to high pressure of arterial circulation results in early, diffuse intimal thickening as a compensatory 

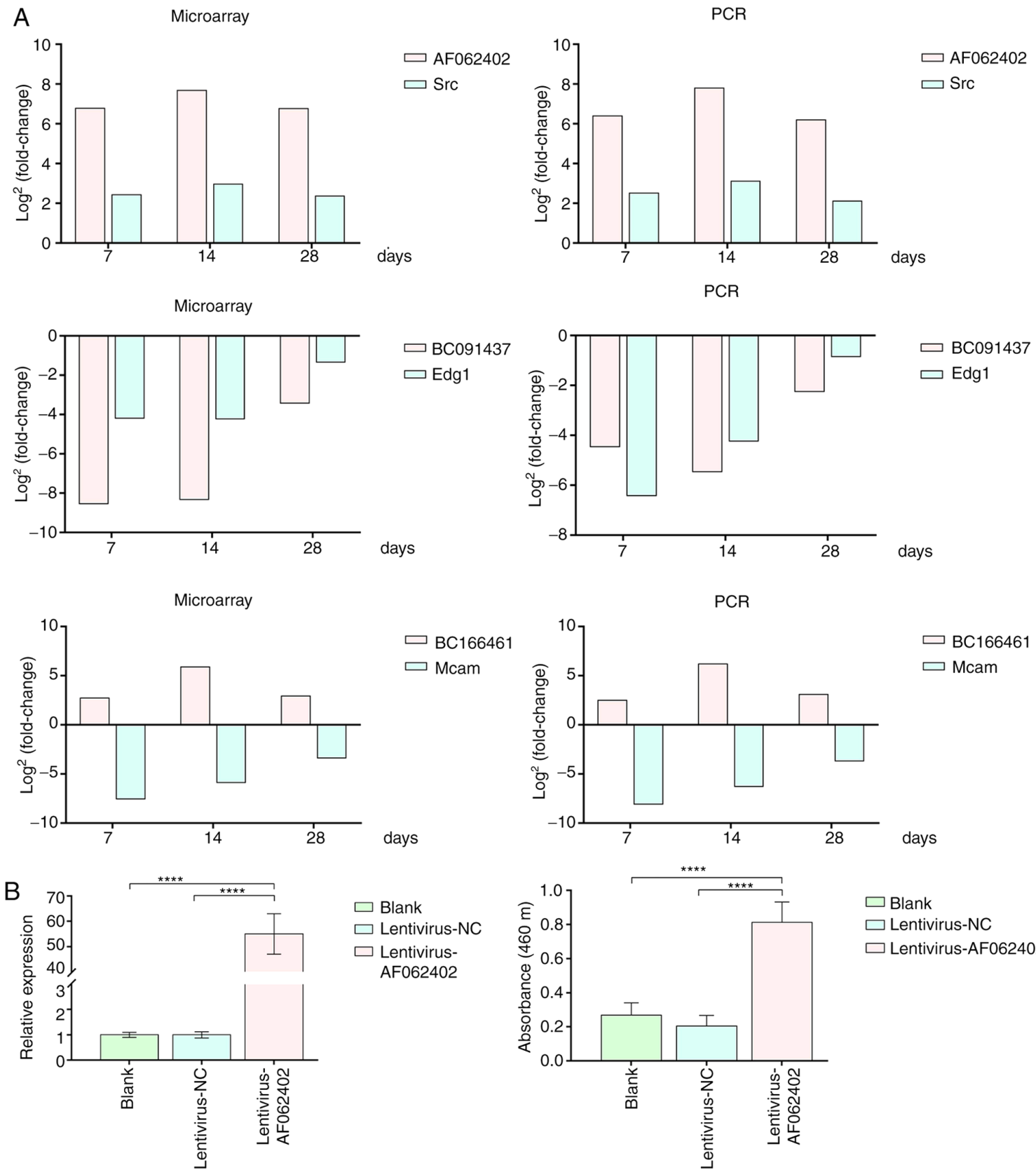

$\square$ Blank

$\square$ Lentivirus-NC

$\square$ Lentivirus-AF062402

Figure 5. Reverse transcription-quantitative PCR validation and Cell Counting Kit-8 assay. (A) Comparison of the expression levels of AF062402, BC091437 and BC166461 and associated protein-coding genes. (B) Expression of AF062402 in the Lentivirus-AF062402 group was significantly higher than that in the blank and Lentivirus-NC groups. Overexpression of AF062402 stimulated the proliferation of vascular smooth muscle cells. ${ }^{* * * * *} \mathrm{P}<0.0001 . \mathrm{NC}$, negative control; Mcam, melanoma cell adhesion molecule.

response termed arterialization (37). With enhanced understanding of the functions of IncRNAs in regulating transcription, IncRNA-mRNA pairs were predicted to be involved in vein graft failure following CABG in the present study. The association between IncRNA and mRNA was the focus of the present study.

As shown in the present study, four protein-coding genes were targeted by both upregulated and downregulated lncRNAs. Edg1, a specific cell surface G-protein coupled receptor, stimulates the activation of multiple cellular events, such as cell proliferation and migration, and the expression of adhesion molecules via S1P signaling (42). c-Src, a member of the Src family, is involved in multiple signaling mechanisms; it not only serves a critical role in mediating the migration and proliferation of VSMCs via the c-Src/protein kinase C (PKC)/MAPK signaling pathway, but is also essential in inducing the expression of Edg1, which is hypothesized to contribute to the progression of intimal thickening following vascular injury in VSMCs $(20,21)$. Overexpression of AF062402 in the present study stimulated the proliferation of VSMCs, verifying the potential therapeutic effect of AF062402-Src in mediating VSMC proliferation. For patients with CABG with diabetes mellitus or hyperlipemia, insulin receptor (Insr) may be a therapeutic target. According to a previous study, overexpression of Insr-A, one of the isoforms of Insr, confers a proliferative advantage on VSMCs in the 
early stages of the atherosclerotic process (43). Furthermore, decreased Insr-A/Insr-B ratio may also contribute to apoptosis of VSMCs and increased risk of atherosclerotic plaque rupture (22). Mcam, an endothelial transmembrane protein of the immunoglobulin superfamily, has been reported to be involved in several important signaling pathways in vascular disease, including PI3K/AKT, p38 and PKC (23). The interaction between Mcam and VEGFR-2 on the endothelial cell surface stimulates cell migration via the AKT and p38 pathways (24). Mcam can also interact with galectin-1 to protect against galectin-1-induced endothelial cell apoptosis (25). Moreover, a previous study revealed a causative role of Mcam in activating lipid-loaded macrophages in plaques, indicating the potential ability of Mcam to be a novel therapeutic target in vascular remodeling following CABG (26).

TGF $\beta-1$ and Fes are two protein-coding genes shown to be targeted by upregulated IncRNAs in the present study. TGF $\beta-1$ is the central regulator of the canonical SMAD2/3 pathway in mediating the deposition of extracellular matrix, which leads to fibrosis in vascular injury (27). A previous study also indicated that TGF $\beta-1$ is involved in regulating the expression of CD40, which serves as the central regulator of various immune responses (28). TGF $\beta$-1 may be responsible for heterogeneous expression of CD40 at the site of vascular bifurcations or curvatures, particularly in the vein grafts of patients with CABG. Fes, one of the known members of a subfamily of the non-receptor tyrosine kinase family, was targeted by 32 upregulated lncRNAs in the present study. Activated Fes kinase has been reported to shift the equilibrium of STAT3, STAT5 and extracellular-signal regulated kinase/MAPK activation to facilitate the adhesion and chemotaxis of inflammatory cells and regulate the proliferation of vascular endothelial cells. Upon phenotypic transition, Fes assists in smooth muscle cell migration to the neo-intima (29).

Yes1 associated transcriptional regulator (Yap) was a protein-coding gene targeted by downregulated lncRNAs in the present study. As a downstream effector, Yap is one of the vital components of the Hippo/Yap pathway, which has been reported to serve roles in mediating vascular restenosis in rodent models (30). Overexpression of Yap combined with intima-media thickness has also been observed in a rat carotid artery balloon injury model (31). In addition, elevated activity of Yap has been reported to mediate the switch of the VSMC phenotype to the synthetic state, promoting neointima formation (32). Moreover, thromboxane A2-specific agonists have been found to induce VSMC migration and proliferation by activating Yap (19). These results demonstrate a potential therapeutic approach for vascular remodeling by activating the Hippo pathway or inhibiting the expression of Yap.

Several limitations of the present study need to be acknowledged. First, since microarray can only detect known gene sequences, certain lncRNAs involved in the vascular remodeling process remain to be investigated. Second, lack of knowledge of the properties and functional mechanisms of lncRNAs involving gene expression meant the role of a specific lncRNA in an overt biological process according to its expression level could not be accurately predicted. Third, although several lncRNAs with potential association in vein graft failure were identified via co-expression network analysis, the detailed mechanisms of these lncRNAs remain unclear; further validation experiments, including western blot analysis, immunohistochemistry and immunofluorescence staining, are required to determine the casual association between these IncRNAs and protein-coding genes overlapped with mRNAs.

In conclusion, the present study provides preliminary evidence of lncRNA expression profiles in vein graft restenosis following vascular grafting. Even though further validation experiments are still required, lncRNAs and target protein-coding genes predicted in the present study may serve as potential therapeutic targets for vein graft restenosis following $\mathrm{CABG}$.

\section{Acknowledgements}

Not applicable.

\section{Funding}

The present study was funded by the National Natural Science Foundation of China (grant nos. 81670327 and 81300155), the Commission of Science Technology of Sichuan Province (grant nos. 2019YJ0046 and 2018SZ0384) and the Clinical Research Incubation Project of West China Hospital of Sichuan University (grant no. 2019HXFH027).

\section{Availability of data and materials}

The datasets generated and/or analyzed during the current study are available in the NCBI Gene Expression Omnibus repository, accession no. GSE152538.

\section{Authors' contributions}

$\mathrm{HZ}$ and JS confirm the authenticity of all the raw data. HZ established the animal model. YZ performed the cell culture experiments. JS and CL analyzed the data and wrote the manuscript. JG and JH contributed to put forward the conception and helped to revise the manuscript. All authors read and approved the final manuscript.

\section{Ethics approval and consent to participate}

The present study was approved by the Animal Ethical and Welfare of Sichuan University, Sichuan, China (approval no. 2018103A).

\section{Patient consent for publication}

Not applicable.

\section{Competing interests}

The authors declare that they have no competing interests.

\section{References}

1. Bhatt DL: CABG the clear choice for patients with diabetes and multivessel disease. Lancet 391: 913-914, 2018.

2. Collet C, Capodanno D, Onuma Y, Banning A, Stone GW, Taggart DP, Sabik J and Serruys PW: Left main coronary artery disease: Pathophysiology, diagnosis, and treatment. Nat Rev Cardiol 15: 321-331, 2018. 
3. Caliskan E, de Souza DR, Böning A, Liakopoulos OJ, Choi YH, Pepper J, Gibson CM, Perrault LP, Wolf RK, Kim KB and Emmert MY: Saphenous vein grafts in contemporary coronary artery bypass graft surgery. Nat Rev Cardiol 17: 155-169, 2020.

4. Aranki SF and Tatooles AJ: Disconnect between vein graft failure and clinical events after coronary artery bypass graft surgery. Circulation 130: 1439-1441, 2014.

5. de Vries MR, Simons KH, Jukema JW, Braun J and Quax PH: Vein graft failure: From pathophysiology to clinical outcomes. Nat Rev Cardiol 13: 451-470, 2016.

6. Petrie MC, Jhund PS, She L, Adlbrecht C, Doenst T, Panza JA, Hill JA, Lee KL, Rouleau JL, Prior DL, et al: Ten-Year outcomes after coronary artery bypass grafting according to age in patients with heart failure and left ventricular systolic dysfunction: An analysis of the extended Follow-Up of the STICH trial (surgical treatment for ischemic heart failure). Circulation 134: 1314-1324, 2016.

7. Escaned J: Secondary revascularization after CABG surgery. Nat Rev Cardiol 9: 540-549, 2012.

8. Lenzen MJ, Boersma E, Bertrand ME, Maier W, Moris C, Piscione F, Sechtem U, Stahle E, Widimsky P, de Jaegere P, et al: Management and outcome of patients with established coronary artery disease: The Euro Heart Survey on coronary revascularization. Eur Heart J 26: 1169-1179, 2005.

9. Brilakis ES, Rao SV, Banerjee S, Goldman S, Shunk KA, Holmes DR Jr,Honeycutt E and Roe MT: Percutaneous coronary intervention in native arteries versus bypass grafts in prior coronary artery bypass grafting patients: A report from the National Cardiovascular Data Registry. JACC Cardiovasc Interv 4: 844-850, 2011.

10. Harskamp RE, Alexander JH, Schulte PJ, Brophy CM, Mack MJ PetersonED,WilliamsJB, Gibson CM,CaliffRM,Kouchoukos NT, etal: Vein graft preservation solutions, patency, and outcomes after coronary artery bypass graft surgery: Follow-up from the PREVENT IV randomized clinical trial. JAMA Surg 149: 798-805, 2014.

11. Fiedler J, Baker AH, Dimmeler S, Heymans S, Mayr M and Thum T: Non-coding RNAs in vascular disease-from basic science to clinical applications: Scientific updatefrom the Working Group of Myocardial Function of the European Society of Cardiology. Cardiovasc Res 114: 1281-1286, 2018.

12. Quinn JJ and Chang HY: Unique features of long noncoding RNA biogenesis and function. Nat Rev Genet 17: 47-62, 2016

13. Halushka PV, Goodwin AJ and Halushka MK: Opportunities for microRNAs in the crowded field of cardiovascular biomarkers. Annu Rev Pathol 14: 211-238, 2019.

14. Wang XW, He XJ, Lee KC, Huang C, Hu JB, Zhou R, Xiang XY, Feng B and Lu ZQ: MicroRNA-221 sponge therapy attenuates neointimal hyperplasia and improves blood flows in vein grafts. Int J Cardiol 208: 79-86, 2016.

15. Garber JC, Barbee RW, Bielitzki JT, Clayton LA, Donovan JC Hendriksen CF, Kohn DF, Lipman NS, Locke PA, Melcher J, et al: Guide for the Care and Use of Laboratory Animals. 8th edition. National Research Council of The National Academies, Washington, DC, 2011.

16. Kuleshov MV, Jones MR, Rouillard AD, Fernandez NF, Duan Q, Wang Z, Koplev S, Jenkins SL, Jagodnik KM, Lachmann A, et al: Enrichr: A comprehensive gene set enrichment analysis web server 2016 update. Nucleic Acids Res 44: W90-W97, 2016

17. Lucaciu O, Băciuţ M, Băciuţ G, Câmpian RS and Ionel A The Gephi Network visualisation of the guided bone regeneration process induced with tissue engineered grafts. In: Key Engineering Materials. Trans Tech Publ 695: 215-221, 2016.

18. Zhao Y, Tang N, Xi D, Huang Z, Zhang T, Liu Y, Wang L, Tang Y, Zhong H and He F: Calcilytic NPS2143 promotes proliferation and inhibits apoptosis of spontaneously hypertensive rat vascular smooth muscle cells via activation of the renin-angiotensin system. Exp Ther Med 20: 818-829, 2020.

19. Feng X, Liu P, Zhou X, Li MT, Li FL, Wang Z, Meng Z, Sun YP, Yu Y, Xiong Y, et al: Thromboxane A2 Activates YAP/TAZ protein to induce vascular smooth muscle cell proliferation and migration. J Biol Chem 291: 18947-18958, 2016.

20. Simo-Cheyou ER, Vardatsikos G and Srivastava AK: Src tyrosine kinase mediates endothelin-1-induced early growth response protein-1 expression via MAP kinase-dependent pathways in vascular smooth muscle cells. Int J Mol Med 38: 1879-1886, 2016

21. Thiel G, Mayer SI, Müller I, Stefano L and Rössler OG: Egr-1-A $\mathrm{Ca}(2+)$-regulated transcription factor. Cell Calcium 47: 397-403, 2010.

22. Beneit N, Martín-Ventura JL, Rubio-Longás C, Escribano Ó, García-Gómez G, Fernández S, Sesti G, Hribal ML, Egido J, Gómez-Hernández A and Benito M: Potential role of insulin receptor isoforms and IGF receptors in plaque instability of human and experimental atherosclerosis. Cardiovasc Diabetol 17: 31, 2018.
23. Wang Z and Yan X: CD146, a multi-functional molecule beyond adhesion. Cancer Lett 330: 150-162, 2013.

24. Jiang T, Zhuang J, Duan H, Luo Y, Zeng Q, Fan K, Yan H, Lu D, Ye Z, Hao J, et al: CD146 is a coreceptor for VEGFR-2 in tumor angiogenesis. Blood 120: 2330-2339, 2012.

25. Colomb F, Wang W, Simpson D, Zafar M, Beynon R, Rhodes JM and Yu LG: Galectin-3 interacts with the cell-surface glycoprotein CD146 (MCAM, MUC18) and induces secretion of metastasis-promoting cytokines from vascular endothelial cells. J Biol Chem 292: 8381-8389, 2017

26. Luo Y, Duan H, Qian Y, Feng L, Wu Z, Wang F, Feng J, Yang D, Qin Z and Yan X: Macrophagic CD146 promotes foam cell formation and retention during atherosclerosis. Cell Res 27: 352-372, 2017.

27. Xie Y, Ostriker AC, Jin Y, Hu H, Sizer AJ, Peng G, Morris AH, Ryu C, Herzog EL, Kyriakides T, et al: LMO7 is a negative feedback regulator of transforming growth factor $\beta$ signaling and fibrosis. Circulation 139: 679-693, 2019.

28. Cooley BC, Nevado J, Mellad J, Yang D, St Hilaire C, Negro A, Fang F, Chen G, San H, Walts AD, et al: TGF- $\beta$ signaling mediates endothelial-to-mesenchymal transition (EndMT) during vein graft remodeling. Sci Transl Med 6: 227ra234, 2014.

29. Greer P: Closing in on the biological functions of Fps/Fes and Fer. Nat Rev Mol Cell Biol 3: 278-289, 2002.

30. He J, Bao Q, Yan M, Liang J, Zhu Y, Wang C and Ai D: The role of Hippo/yes-associated protein signalling in vascular remodelling associated with cardiovascular disease. Br J Pharmacol 175: 1354-1361, 2018

31. Ono H, Ichiki T, Ohtsubo H, Fukuyama K, Imayama I, Hashiguchi Y, Sadoshima J and Sunagawa K: Critical role of Mst1 in vascular remodeling after injury. Arterioscler Thromb Vasc Biol 25: 1871-1876, 2005.

32. Wang X, Hu G, Gao X, Wang Y, Zhang W, Harmon EY, Zhi X, Xu Z, Lennartz MR, Barroso M, et al: The induction of yes-associated protein expression after arterial injury is crucial for smooth muscle phenotypic modulation and neointima formation. Arterioscler Thromb Vasc Bio 32: 2662-2669, 2012.

33. Wu G, Cai J, Han Y, Chen J, Huang ZP, Chen C, Cai Y, Huang H, Yang Y, Liu Y, et al: LincRNA-p21 regulates neointima formation, vascular smooth muscle cell proliferation, apoptosis, and atherosclerosis by enhancing p53 activity. Circulation 130: 1452-1465, 2014

34. Wang Z, Zhang XJ, Ji YX, Zhang P, Deng KQ, Gong J, Ren S, Wang X, Chen I, Wang H, et al: The long noncoding RNA Chaer defines an epigenetic checkpoint in cardiac hypertrophy. Nat Med 22: 1131-1139, 2016

35. Yahagi K, Kolodgie FD, Otsuka F, Finn AV, Davis HR, Joner M and Virmani R: Pathophysiology of native coronary, vein graft, and in-stent atherosclerosis. Nat Rev Cardiol 13: 79-98, 2016.

36. Storey RF: Exploring mechanisms of graft occlusion toward improved outcomes in coronary artery bypass graft surgery. J Am Coll Cardiol 57: 1078-1080, 2011.

37. Jelaković B, Nikolić J, Radovanović Z, Nortier J, Cosyns JP, Grollman AP, Bašić-Jukić N, Belicza M, Bukvić D, Čavaljuga S, et al: Consensus statement on screening, diagnosis, classification and treatment of endemic (Balkan) nephropathy. Nephrol Dial Transplant 29: 2020-2027, 2014.

38. Sabik JF II: Understanding saphenous vein graft patency. Circulation 124: 273-275, 2011

39. Lee R, Margaritis M, Channon KM and Antoniades C: Evaluating oxidative stress in human cardiovascular disease: Methodological aspects and considerations. Curr Med Chem 19: 2504-2520, 2012.

40. Tóth A, Balogh E and Jeney V: Regulation of vascular calcification by reactive oxygen species. Antioxidants (Basel) 9: 963, 2020.

41. Muslin AJ: MAPK signalling in cardiovascular health and disease: Molecular mechanisms and therapeutic targets. Clin Sci (Lond) 115: 203-218, 2008.

42. Garcia JG, Liu F, Verin AD, Birukova A, Dechert MA, Gerthoffer WT, Bamberg JR and English D: Sphingosine 1-phosphate promotes endothelial cell barrier integrity by Edg-dependent cytoskeletal rearrangement. J Clin Invest 108: 689-701, 2001.

43. Ullrich A, Gray A, Tam AW, Yang-Feng T, Tsubokawa M, Collins C, Henzel W, Le Bon T, Kathuria S, Chen E, et al: Insulin-like growth factor I receptor primary structure: Comparison with insulin receptor suggests structural determinants that define functional specificity. EMBO J 5: 2503-2512, 1986.

This work is licensed under a Creative Commons Attribution-NonCommercial-NoDerivatives 4.0 International (CC BY-NC-ND 4.0) License. 